\title{
Increased incidence of urinary tract infection in patients with rheumatoid arthritis and secondary Sjögren's syndrome
}

\author{
Moshe Tishler, Dan Caspi, Yehoshua Almog, Rafael Segal, Michael Yaron
}

\begin{abstract}
The incidence of lower urinary tract infection in $\mathbf{1 2 0}$ women with rheumatoid arthritis and secondary Sjögren's syndrome was evaluated retrospectively. Thirty one patients $(26 \%)$ had secondary Sjögren's syndrome. Recurrent urinary tract infection was significantly more common in these patients (11/31) than in patients without Sjögren's syndrome (4/89). Habitual leucocyturia was also more common in patients with secondary Sjögren's syndrome (18/31) than in patients with rheumatoid arthritis without Sjögren's syndrome (8/89). Of seven patients with vaginal sicca symptoms, six had recurrent urinary tract infection. Urinary 24 hour mucopolysaccharide excretion in 20 patients with Sjögren's syndrome was similar to the excretion in 10 patients without Sjögren's syndrome. These results show that recurrent urinary tract infection is significantly more common in women with rheumatoid arthritis and secondary Sjögren's syndrome. A local deficit in protective urinary mucosal secretion or other immune mechanisms may be responsible for this susceptibility.
\end{abstract}

Sjögren's syndrome is a chronic autoimmune disorder with various clinical presentations. Extraglandular manifestations of the disease may include focal or diffuse lymphocytic infiltration in different tissues and a variety of autoimmune phenomena. In primary Sjögren's syndrome renal disease is well documented and consists primarily of latent or overt interstitial disease. ${ }^{1}$ In patients with secondary Sjögren's syndrome, however, renal disease is usually not related to Sjögren's syndrome and develops as a result of the underlying autoimmune disorder. Infection of the lower urinary tract in either primary or secondary Sjögren's syndrome has not been reported previously. Following patients with rheumatoid arthritis, we had the clinical impression that women with Sjögren's syndrome had frequent infections of the lower urinary tract. The purpose of this study was to evaluate the incidence of urinary tract infection in patients with rheumatoid arthritis and secondary Sjögren's syndrome.

Patients and methods

One hundred and twenty women with rheumatoid arthritis diagnosed according to the American Rheumatism Association criteria, ${ }^{2}$ attending our outpatient clinic, were randomly included in the study. A detailed questionnaire on the symptoms and signs of Siögren's syndrome in several systems was completed. Patients were asked specifically about urinary and vaginal manifestations, episodes of urinary tract infection treated in other hospitals, drug allergies, and the use of other drugs which might cause dryness of the mucosal membrane. Urinary tract infection was diagnosed by the clinical symptoms of frequency, dysuria, and urgency associated with a bacterial culture of more than 100000 colonies per millilitre and pyuria (more than five white blood cells per high power field). Schirmer's test was performed without anaesthesia on all patients. ${ }^{3}$ Patients with a positive (less than $5 \mathrm{~mm}$ ) or borderline test (between 5 and $10 \mathrm{~mm}$ ) but definite sicca symptoms were examined ophthalmologically, including break up time, tear level, and a slit lamp examination. ${ }^{3}$

Urine analysis, including $\mathrm{pH}$, osmolality, and direct microscopy, was performed. Twenty four hour excretion of urinary proteins, electrolytes, calcium, and phosphorus was measured, and the creatinine clearance test was performed in all patients. Urinary mucopolysaccharides were evaluated according to a method described by Anderson and Machagan. ${ }^{4}$

Diagnosis of secondary Sjögren's syndrome in our patients was based on the criteria proposed by Daniels and Talal ${ }^{5}$ and included: (a) typical symptoms of xerostomia and xerophthalmia; (b) keratoconjunctivitis sicca diagnosed by a pathological Schirmer's test, characteristic slit lamp examination, and abnormal break up time; and (c) existence of another collagen disease (rheumatoid arthritis).

\section{Results}

The table gives the characteristics of the 120 patients with rheumatoid arthritis studied. Thirty one patients $(26 \%)$ had secondary Sjögren's syndrome. There were no statistical differences in age, disease duration, and presence of rheumatoid factor between the 31 patients with secondary Sjögren's syndrome and 89

Characteristics of 120 patients with rheumatoid arthritis studied

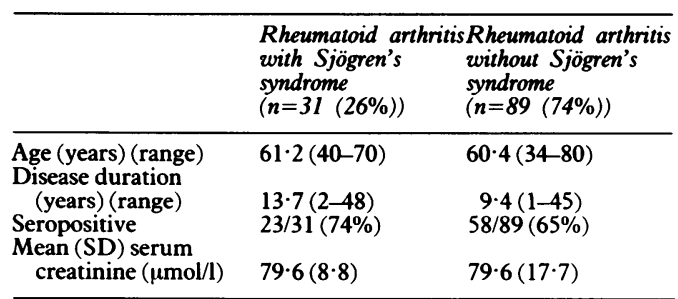


patients with rheumatoid arthritis without Sjögren's syndrome, nor were there differences between the two groups in the use of corticosteroids or immunosuppressive drugs such as azathioprine and methotrexate. Functional capacity was similar in the two groups and no patient could be classified as functional class IV. Recurrent urinary tract infection was defined as three or more episodes of urinary infection per year which were diagnosed clinically and by urine analysis and treated by antibiotics. Recurrent urinary tract infection was significantly more common in patients with rheumatoid arthritis and secondary Sjögren's syndrome (11/31) than in patients with rheumatoid arthritis without Sjögren's syndrome (4/89) $(p<0.001)$. Habitual leucocyturia was defined as a persistent finding of more than 10 white blood cells per high power field and was significantly more common in patients with secondary Sjögren's syndrome (18/31) compared with patients with rheumatoid arthritis without Sjögren's syndrome (8/89) $(p<0.001)$ (fig 1$)$. Recurrent urinary tract infection was not associated with higher morbidity in respiratory tract infections.

Vaginal sicca symptoms were reported by only seven of 31 patients with Sjögren's syndrome, six of whom also had recurrent urinary tract infection. Urinary $\mathrm{pH}$, osmolality, and electrolyte excretion were normal in all 31 patients with Sjögren's syndrome. Twenty patients were chosen randomly and evaluated for urinary excretion of mucopolysaccharides. There were no differences in urinary excretion of mucopolysaccharides between 10 patients with secondary Sjögren's syndrome and 10 patients without sicca syndrome.

Data for the infecting organisms are not fully available as part of the infective episodes were treated in the community. The most common organism recovered in urinary cultures available to us was Escherichia coli.
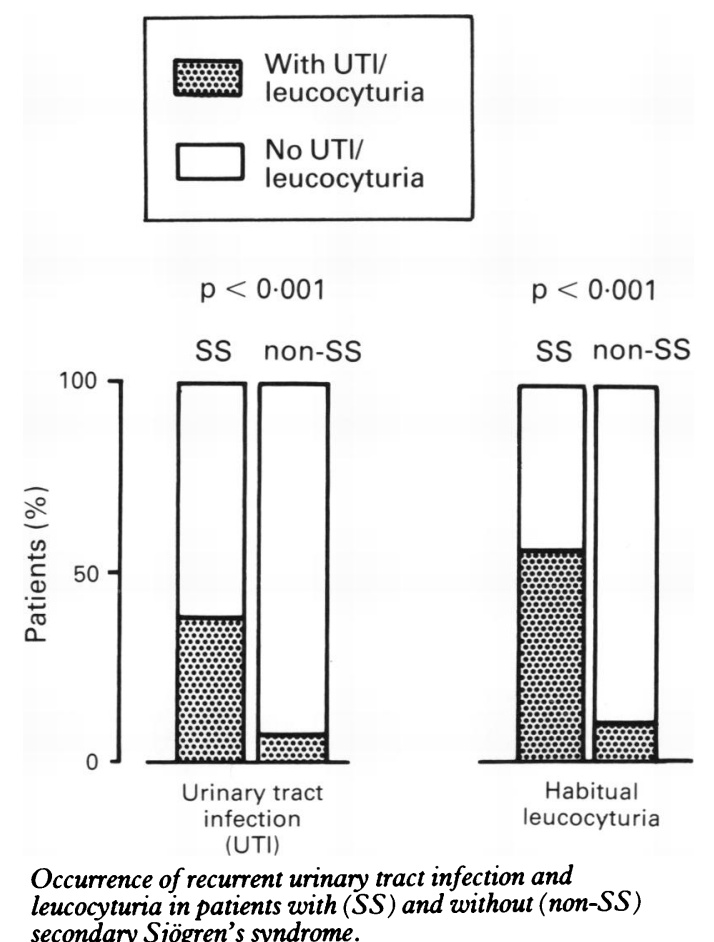

\section{Discussion}

Dryness of mucosal membranes in Sjögren's syndrome has been associated with frequent infections of the affected organs. The dry eye is at a higher risk of superficial infection, probably because of the loss of the lubricant and antibacterial activities of tears. ${ }^{6}$ Oral and vaginal mucosa were found to be infected by candida species $^{7}$ whereas the frequency of recurrent respiratory infection is under debate. ${ }^{89}$

Interstitial nephritis has been reported as the most common nephrological feature in primary Sjögren's syndrome, ${ }^{1}$ usually manifested by type I renal tubular acidosis. Furthermore, renal function abnormalities were found to be confined to distal and proximal tubules. ${ }^{10}$ Although renal tubular acidosis could be detected in $22-30 \%$ of these patients, ${ }^{11}$ urinary findings have been unimpressive and the occurrence of urinary infections has not been described. In patients with secondary Sjögren's syndrome the situation is more complicated, as renal disease is typically related to the underlying disease process-for example, lupus nephritis, amyloidosis, or scleroderma kidney. The group of women patients with rheumatoid arthritis described here could be said to have 'sicca complex' rather than Sjögren's syndrome, as our criteria were not identical to those proposed by the California or the Copenhagen groups. ${ }^{12} 13$ Nevertheless we feel that considering the clinical symptoms and findings, it is likely that these patients did have secondary Sjögren's syndrome.

Our finding of an association between recurrent urinary tract infection and secondary Sjögren's syndrome has not been described before. We do not have a clear explanation for this observation. As the use of corticosteroids and immunosuppressive drugs was similar in the two groups, it would not seem to increase the rate of infection in patients with Sjögren's syndrome. We suspect that a deficit in protective mucosal secretions in the lower urinary tract may contribute to the susceptibility to infection. To evaluate the mucus content of the urinary tract, excretion of mucopolysaccharides in urine (which reflects local and systemic production of mucopolysaccharides) was measured. Nevertheless, differences between patients with Sjögren's syndrome and urinary tract infection and patients with rheumatoid arthritis without Sjögren's syndrome or urinary tract infection could not be detected. The presence of recurrent urinary tract infection in six of seven patients with vaginal dryness may further direct attention towards a local predisposing factor. The possibility of mucosal atrophy cannot be ruled out as a trigger for infection, as such atrophy has been described in other organs, such as the gastric mucosa. ${ }^{14}$

Another possible explanation for our clinical observation might be connected with secretory IgA levels in urine. Several papers have reported lower levels of secretory IgA in women with recurrent urinary tract infection and postulated that the deficient production of $\operatorname{IgA}$ increases the susceptibility of these women to infections. ${ }^{15}$

This observation of increased occurrence of urinary tract infection in patients with secondary 
Sjögren's syndrome increases the number of systems affected by this syndrome and calls for further study.

1 Shearn M, Tu W. Nephrogenic diabetes insipidus and other defects of renal tubular function in Sjögren's syndrome. $A m$ f Med 1965; 39: 312-8.

2 Arnett F C, Edworthy S, Block D A, et al. The 1987 revised ARA criteria for the classification of rheumatoid arthritis. Arthritis Rheum 1988; 31: 315-24.

3 Lamberts D W. Keratoconjunctivitis sicca. In: Smolin G, Thoft R A, eds. The cornea. Boston: Little Brown, 1983: 293-309.

4 Anderson A J, Machagan N F. The isolation and estimation of urinary mucoproteins. Biochem f 1955; 59: 638-44.

5 Daniels T E, Talal N. Diagnosis and differential diagnosis of Sjögren's syndrome. In: Talal N, Moutsopoulos H M, Kassah S S, eds. Sjögren's syndrome. Berlin: Springer Verlag, 1987: 193-9.

6 Lamp M A, Gold J B. An in vivo study of the corneal surface in keratoconjunctivitis sicca. Trans Ophthalmol Soc UK $1985 ; 104: 436-8$.

7 Holmstrup P, Besserman M. Clinical, therapeutic and pathogenic aspects of chronic oral multifocal candidiasis. Oral Surg 1983; 56: 388-95.

8 Shearn M. Sjögren's syndrome. Med Clin N Am 1977; 61 271-82.

9 Papathanasious M P, Constantopoulos S H, Tsampoulas C, Orosos A A, Moutsopoulos H M. Reappraisal of respiratory abnormalities in primary and secondary Sjögren's syndrome: a controlled study. Chest 1986; 90: 370-94.

10 Shiozawa S, Shiozawa K, Shimizu S, Makaoa M, Isobe T, Fujita T. Studies of renal disease in Sjögren's syndrome. Ann Rheum Dis 1987; 46: 768-72.

11 Talal N, Zisnan E, Schur P. Renal tubular acidosis glomerulonephritis and immunologic factors in Sjögren's syndrome. Arthritis Rheum 1968; 11: 774-86.

12 Manthorpe R, Oxholm P, Prause J U, Schiødt M. The Copenhagen criteria for Sjögren's syndrome. Scand $\mathcal{F}$
Rheumatol Suppl 1980; 61: 19-21.

13 Fox R I, Robinson C A, Curo J G, Kozin F, Howell F V. Sjögren's syndrome: Proposed criteria for classification. Arthritis Rheum 1986; 29: 577-85.

14 Kilpi A, Bergroth V, Konttinen Y T, Maury C P J, Reitamo $S$, Wegelius $O$. Lymphocytic infiltration of the gastric mucosa in Sjögren's syndrome. Arthritis Rheum 1983; 26: 1196-200.

15 Riedasch G, Hech P, Rauterberg E, Ritz E. Does low urinary sIgA predispose to urinary tract infection? Nephrol Int 1983; 23: 75-6. 